\title{
COMPARAÇÃO ENTRE ATIVIDADES DE TREINO PARA O APRIMORAMENTO DA FINALIZAÇÃO NO FUTSAL
}

\author{
Marcelo Figueiró Baldi ${ }^{1}$
}

BALDI, M. F. Comparação entre atividades de treino para o aprimoramento da finalização no futsal. Arq. Cienc. Saúde UNIPAR, Umuarama, v. 24, n. 2, p, 101-104, maio/ago. 2020.

\begin{abstract}
RESUMO: Existem diferentes atividades de treino que o treinador pode optar para o desenvolvimento da finalização na iniciação ao futsal. Contudo, são escassos os estudos que, de fato, trazem informações científicas que possam subsidiar o treinador a fazer as melhores escolhas. Diante do exposto, o presente estudo teve como objetivo comparar diferentes atividades de treino para o desenvolvimento da finalização na iniciação ao futsal. Participaram do estudo 23 jogadores de 8 a 11 anos de uma escola de futsal do noroeste do Paraná. Foram avaliadas as atividades: a) jogo formal, b) jogo reduzido, c) gol a gol e d) chute a gol. Os resultados apontam que, o "gol a gol” é a atividade que proporciona a maior média de finalização. Por outro lado, foi na atividade de chute a gol em que se observou o maior percentual de acertos das finalizações e menor diferença na frequência de chutes com a perna dominante e não dominante. Por fim, foi observado que nenhuma das atividades proporcionou exercitação suficiente da finalização com outras partes do corpo.
\end{abstract}

PALAVRAS-CHAVE: Criança. Aptidão. Educação física e treinamento.

\section{COMPARISON BETWEEN TRAINING ACTIVITIES TO IMPROVE FINALIZATION IN FUTSAL}

\begin{abstract}
There are different training activities that the coach can choose for the development of the finalization in the initiation to futsal. However, there are few studies that actually provide scientific information that can help the coach make the best choices. Therefore, this study aimed at comparing different training activities for the development of the finalization in the futsal initiation. A total of 23 players aged from 8 to 11 from a futsal academy in northwestern Paraná took part in this study. The following activities were evaluated: a) formal game; b) small-sided game; c) goal to goal; and d) shot at goal. The results indicate that the goal-to-goal is the activity that provides the highest average of finishing among them. On the other hand, the highest percentage of correctness of finalizations was observed in the shot-at-goal activity, as well as the smallest difference in the frequency of kicks with the dominant and non-dominant leg. Finally, it was observed that none of the activities provided sufficient stimulation to other parts of the body.
\end{abstract}

KEYWORDS: Child. Aptitude. Physical education and training.

Introdução

No futsal existem diferentes tipos de atividades que podem ser utilizadas como ferramentas para o desenvolvimento da finalização em crianças (GARGANTA, 2002). De acordo com Baldi (2018) a finalização compreende toda tentativa de enviar a bola em direção à meta com o intuito de efetuar um gol.

Dentre as principais atividades utilizadas para o treinamento da finalização, nos clubes e escolinhas de futsal, destacam-se o próprio jogo formal, jogos reduzidos, atividades lúdicas da cultura infantil e atividades analíticas (ZISKIND, 2011). A saber, jogo formal nada mais é do que o jogo oficial, com suas regras bem definidas. Por exemplo, no futsal o jogo formal se joga 5 contra 5 em uma quadra com dimensões pré-estabelecidos pelas regras oficias do jogo.

Por outro lado, jogos reduzidos são jogos que mantém a estrutura funcional do jogo formal, mas são modificados principalmente no que diz respeito ao número de jogadores participantes e as dimensões da quadra. Por exemplo, ao invés de jogar 5 contra 5, jogam 3 contra 3 em uma quadra com dimensões menores. Em geral, este tipo de atividade é muito utilizado no dia a dia do treinamento da iniciação ao futsal, tendo em vista que, proporciona aos jogadores uma participação mais efetiva no jogo (BALDI, 2014).

Outra tipo de atividade que pode ser incluída no processo de ensino, aprendizagem e treinamento da finalização no futsal é o "gol a gol” (SCAGLIA, 2003). Tal atividade tem origem na cultura lúdica infantil e pode ser perfeitamente encaixada dentro dos processos de formação
(SCAGLIA, 2003).

Ainda, existem as atividades analíticas tal como o chute ao gol. Este tipo de atividade geralmente exclui toda a imprevisibilidade do jogo, trabalhando a finalização de forma isolada em ambiente altamente controlado, frequentemente sem a influência de fatores externos (LEITÃO, 2009).

Por fim, é importante destacar que cada tipo de atividade apresenta suas vantagens e desvantagens e que todas constituem-se como ferramentas que podem ser úteis no processo de desenvolvimento da finalização no futsal (BALDI, 2014).

Contudo, apesar da riqueza e coerência das proposições teóricas a respeito destes diferentes tipos de atividades (GRAÇA, 1998; ZISKIND, 2011), de nosso conhecimento não existem estudos científicos que tenham comparado o impacto destas atividades na média, efetividade (percentual de acertos) e formas de execução da finalização em crianças de 8 a 11 anos de idade de escolinhas de futsal. Desta forma, perpetua-se o distanciamento entre teoria e prática deixando os treinadores sem a evidência científica para subsidiar as suas práticas profissionais com jogadores desta faixa etária no futsal.

Diante do exposto, o presente estudo tem como intenção responder a seguinte questão: existe diferença na média, percentual de acertos e formas de execução da finalização entre jogo formal, jogo reduzido, "gol a gol" e "chute à gol” em crianças de 8 a 11 anos praticantes de futsal? 


\section{Materiais e Métodos}

\section{Caracterização do estudo}

Esta pesquisa caracteriza-se como um estudo de caso, aplicado, quantitativo e de campo (THOMAS; NELSON; SILVERMAN, 2012).

\section{Participantes}

Participaram do estudo 23 jogadores de futsal, do sexo masculino, de 8 a 11 anos, de uma escola de futsal do noroeste do Paraná. Os indivíduos treinam 2 vezes por semana ( \pm 1 hora por sessão) as quartas e sextas-feiras.

\section{Procedimentos}

Após obter o parecer favorável do Comitê de Ética em Pesquisa com Seres Humanos da Universidade Paranaense (CAEE, 71943717.8.0000.0109) os pais foram contatados por meio de um termo de consentimento livre e esclarecido para a participação dos seus filhos na pesquisa. Neste documento, foram apresentadas a proposta do estudo, esclarecidos os objetivos e métodos empregados. $\mathrm{O}$ estudo teve início somente após obter o consentimento de todos os envolvidos. Todas as avaliações foram realizadas em um prazo de 15 dias, no período da tarde no próprio ginásio de treino da escolinha.

\section{Protocolo de avaliação}

Para comparar a média, percentual de acertos e formas de realização da finalização nas atividades de jogo formal, jogo reduzido, "gol a gol" e "chute ao gol, os jogadores foram filmados durante as diferentes atividades. A câmera foi posicionada a 3 metros da linha lateral e a 4 metros de altura. Todos as atividades tiveram uma duração de 20 minutos contínuos sem interrupção. Todos os participantes da pesquisa já tinham experiência com as atividades de treino. As coletas foram conduzidas em 4 sessões de treinos diferentes e na seguinte sequência: jogo formal, jogo reduzido, gol a gol e chute ao gol.

Em todas as atividades, os treinadores (estagiários acadêmicos de Educação Física) participaram ativamente podendo instruir os jogadores. Contudo, foi solicitado não interromper a atividade.

Nas atividades-jogo as equipes foram equilibradas de forma competitiva mediante a avaliação subjetiva dos treinadores, conforme realizam, normalmente, em uma sessão de treinamento.

\section{a) Jogo formal}

O jogo formal consiste de 2 equipes de 5 jogadores cada (sendo 1 goleiro e 4 jogadores de "linha". As equipes jogaram entre si de acordo com as regras oficiais do futsal.

\section{b) Jogo reduzido}

O jogo reduzido consiste de 2 equipes de 3 jogadores cada (sendo 1 goleiro e 2 jogadores de "linha"). O tamanho da quadra foi ajustado proporcionalmente ao número de jogadores envolvidos. Para tornar o jogo mais dinâmico e com menos interrupções, a equipe ao sofrer um gol reiniciava as suas ações ofensivas automaticamente com a reposição do goleiro. c) "Gol a gol"

Nesta atividade jogaram 2 crianças (uma contra a outra). A atividade de gol a gol é um jogo da cultura lúdica infantil que consiste em fazer gol na meta adversária e não sofrer gol em sua própria meta. Para isso, cada criança em sua metade do campo se alterna em chutar a bola em direção à meta adversária. Ganha a criança que ao final dos 20 minutos fizer mais gols.

\section{d) Chute ao gol}

O chute a gol é uma atividade analítica na qual a criança passa a bola ao professor que "ajeita" a bola de volta para a criança que vem na corrida e chuta ao gol. Nesta atividade, as crianças aguardavam em fila a sua vez de finalizar ao gol.

\section{Procedimento de análise dos dados}

Três observadores treinados analisaram, classificaram e registraram as ações técnicas de finalização em todas as atividades. Os observadores realizaram todas as análises em conjunto. As ações que geraram divergências entre os observadores foram discutidas até chegar a um consenso. Os procedimentos de análise foram seguidos de acordo com a proposta de Blomqvist, Vanttinen e Luhtanen (2005).

\section{Resultados e Discussão}

Nos Gráficos 1 a 3 são apresentados os resultados referentes a média, efetividade e formas de execução da finalização em jogo formal, jogo reduzido, gol a gol e chute a gol em crianças de 8 a 11 anos de idade de uma escolinha de futsal do noroeste do Paraná. Especificamente, no Gráfico 1 são apresentados os resultados referentes à média de finalização por jogador. No Gráfico 2 são apresentados os resultados referentes à efetividade da finalização. E no Gráfico 3 são apresentados os resultados referentes às formas de execução da finalização.

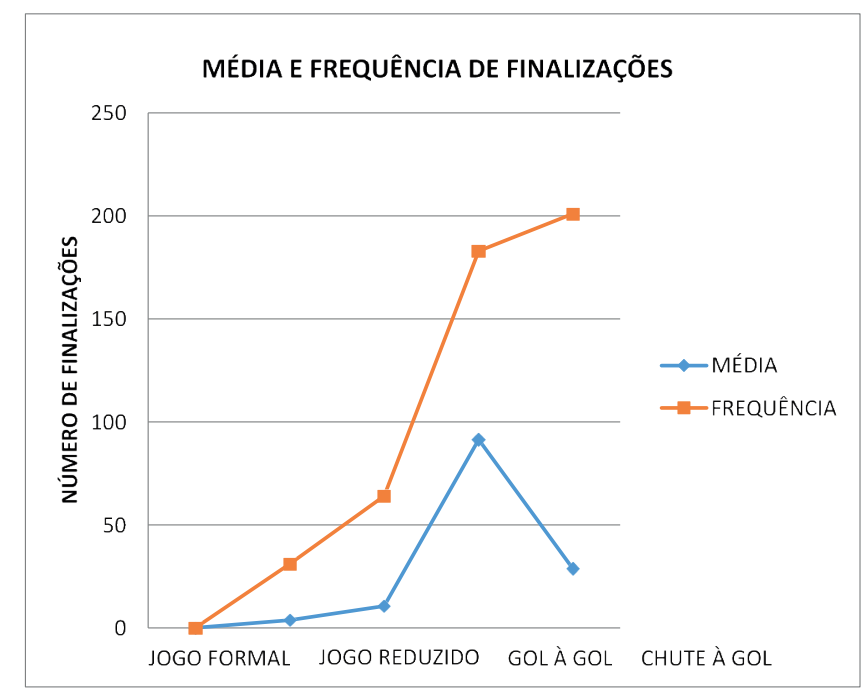

Gráfico 1: Média e frequência de finalizações em jogo formal, jogo reduzido, gol a gol e chute à gol em crianças de 8 a 11 anos de idade de uma escolinha de futsal do noroeste do Paraná $(\mathrm{n}=23)$. 
Conforme apresentado no gráfico 1, percebe-se que o "chute a gol" apresentou a maior frequência total de finalizações. Contudo, foi no jogo "gol a gol" em que se percebeu a maior média de finalizações por jogador. Isso justifica-se pelo fato deste jogo possuir apenas 2 jogadores participantes enquanto que na atividade de chute à gol haviam 6 atletas, em fila, esperando por sua vez para finalizar.

Os resultados do gol a gol destacam-se e alinhamse às recomendações da literatura científica que sugere atividades que proporcionam participação efetiva dos jogadores e maiores possibilidades de concretização (GRAÇA, 1998). Pode-se perceber também que, por outro lado, estes dados corroboram observações prévias de que conforme o aumento do número de jogadores na atividade, restringe progressivamente a participação dos jogadores e consequentemente a consecução das ações técnicas relevantes ao objetivo da atividade (BALDI, 2014).

No outro extremo, isto é, em jogo formal, dada a maior dificuldade e complexidade da atividade (LEITÃO, 2009) observou-se a menor frequência e média de finalização. A que se levar em conta também que nesta atividade existem mais paralizações devido à faltas, cobranças de laterais e escanteios. Em relação a isso, Lima (2010) indica que, em diferentes atividades, em especial, atividades que utilizam mais jogadores há mais embates e intervenções diretas sobre a bola, e assim uma menor continuidade das ações. Portanto, diante destes achados pode-se concluir que a atividade que proporciona maior média de finalização é o "gol a gol".

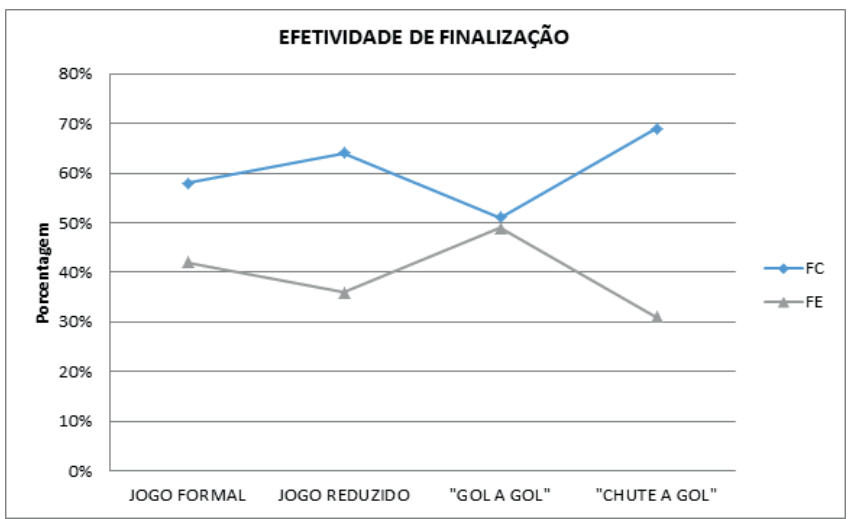

Gráfico 2: Efetividade da finalização em jogo formal, jogo reduzido, gol a gol e chute a gol em crianças de 8 a 11 anos de idade de uma escolinha de futsal do noroeste do Paraná $(\mathrm{n}=23)$.

No gráfico 2 pode-se observar que no "chute a gol" foi observado o maior percentual de acertos da finalização comparado as demais atividades. Por outro lado, nota-se que o gol a gol foi a atividade que demonstrou o menor percentual de acertos. De fato, dada as suas características, a atividade de chute a gol é a que apresenta a menor complexidade e dificuldade (BALDI, 2018). Tais resultados justificamse dada a sua característica analítica (LEITÃO, 2009; ZISKIND, 2011). Muito diferentes de atividades dinâmicas e complexas que carregam os elementos sistêmicos do jogo (GRÉHAIGNE, 1997; DAVIDS et al., 2013). Em síntese, o chute a gol foi a atividade que proporcionou aos jogadores maior percentual de acertos nas finalizações.

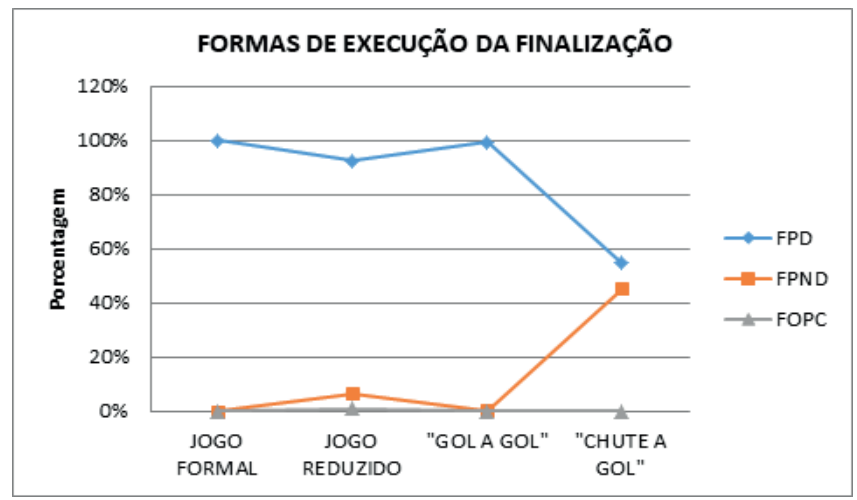

Gráfico 3. Formas de realização da finalização em jogo formal, jogo reduzido, gol a gol e chute a gol em crianças de 8 a 11 anos de idade de uma escolinha de futsal do noroeste do Paraná $(\mathrm{n}=23)$.

Legenda: FPD = finalização com a perna dominante; FPND = finalização com a perna não dominante; $\mathrm{FOPC}$ = finalização com outras partes do corpo.

No gráfico 3 é possível observar que em todos os jogos houve uma predominância do uso da perna dominante para a realização da finalização. Em especial, o jogo formal e "gol a gol" foram os jogos que apresentaram a maior utilização da perna dominante. No jogo reduzido houve uma pequena utilização da perna não dominante. Somente no chute a gol foi registrada a realização de finalização mais equilibrada entre perna dominante e perna não dominante. Diante disso, é possível afirmar que, exceto na atividade de chute à gol, os demais jogos não estimulam a utilização da perna não-dominante.

A não utilização da perna não-dominante, exceto na atividade de chute à gol, justifica-se pelo fato de que todo o gesto de realização correta da técnica, acarreta um desempenho de forma econômica e objetiva (FLÔRES, 2011). Por isso, foi observado que as crianças, quando tem a autonomia de decisão, acabam por optar por realizar finalizações com sua perna dominante, visto que elas se sentem mais confiantes e seguras. Para além destas discussões, observou-se que nenhuma das atividades estimula finalizações com outras partes do corpo.

Em síntese, corroborando dados de pesquisas anteriores (BALDI, 2018), os jogos investigados não se mostraram eficientes para estimular a finalização executada com outras partes do corpo e apenas o chute a gol proporciona a estimulação da perna não-dominante. Desta forma, ao se optar por estes jogos, dentro destas configurações, recomenda-se a utilização do chute à gol para o desenvolvimento bilateral e modificações das regras ou até mesmo outros jogos/atividades, se o objetivo do treinador for desenvolver a finalização com outras partes do corpo.

\section{Conclusão}

Ao comparar jogo formal, jogo reduzido, gol a gol e chute a gol para o aprimoramento da finalização em crianças de 8 a 11 anos de idade de uma escolinha de futsal do noroeste do Paraná, é possível concluir que, o "gol a gol” foi a atividade que proporcionou a maior média de finalização. Por outro lado, foi na atividade de chute a gol em que se 
observou o maior percentual de acertos das finalizações bem como a menor diferença nos chutes utilizando a perna dominante e não-dominante. Por fim, foi observado que nenhuma das atividades proporcionou exercitação suficiente da finalização com outras partes do corpo.

\section{Referências}

BALDI, M. F. Treinamento tático no futebol: efeito das modificações de jogos reduzidos. 2014. 93 f. Dissertação (Mestrado em Educação Física) - Universidade Federal de Santa Catarina, 2014.

BALDI, M. F. et al. Efeito do número de jogadores na frequência e distribuição dos fundamentos técnicos em jogos reduzidos na iniciação ao futebol. Arquivos de Ciências da Saúde da Unipar, v. 22, n. 1, p. 27-32, 2018.

BLOMQVIST, M.; VANTTINEN, T.; LUHTANEN, P. Assessment of secondary school students' decision-making and game-play ability in soccer. Physical Education and Sport Pedagogy, v. 10, n. 2, p. 107-119, 2005.

DAVIDS, K. et al. How small-sided and conditioned games enhance acquisition of movement and decision-making skills. Exercise and Sport Sciences Review, v. 41, n. 3, p. 154-161, 2013.

FLÔRES, F. S. Fundamentos Técnicos: a base do futsal. EFDeportes.com, Revista Digital. Buenos Aires, v. 16, n. 163 , dezembro de 2011 .

GARGANTA, J. Competências no ensino e treino de jovens futebolistas. Revista Digital. Buenos Aires, ano 8, n. 45, Fevereiro. 2002.

GRAÇA, A. Os comos e os quandos no ensino dos jogos. In: GRAÇA, A.; OLIVEIRA, J. O ensino dos jogos desportivos. Porto: Rainho \& Neves, 1998, p. 27-34.

GRÉHAIGNE, J. F.; BOUTHIER, D.; DAVID, B.

Dynamic-system analysis of opponent relationships in collective actions in soccer. Journal of Sports Science, v. 15, n. 2, p. 137-149, 1997.

LEITÃO, R. A. O jogo de futebol: investigação de sua estrutura, seus modelos e da inteligência de jogo, do ponto de vista da complexidade. 2009. 244 f. Tese de doutorado. Faculdade de Educação Física da Unicamp, Campinas, 2009.

LIMA, N. J. C. Jogos reduzidos em futebol:

Comportamento Técnico-Táctico e Variabilidade da Frequência Cardíaca em Jogos de 3x3 e 6x6 com Jogadores Sub-13. 2010. 47 f. Dissertação (mestrado em ciências do desporto: Especialização em jogos desportivos colectivos) - Universidade de Trás-os-Montes e Alto Douro, Vila Real, 2010.

SCAGLIA, A. J. O futebol e os jogos/brincadeiras de bola com os pés: todos semelhantes, todos diferentes. Tese de doutorado. Faculdade de Educação Física da Unicamp, Campinas, 2003.

THOMAS, J. R.; NELSON, N.; SILVERMANN, S. J. Métodos de pesquisa em atividade física. 6 . ed. Porto Alegre: Artmed, 2012.

ZISKIND. F. S. Jogos reduzidos e adaptados no futebol. Universidade do Futebol. Disponível em: http://www. universidadedofutebol.com.br. Acesso em: 01 nov. 2018.

\section{Nota do autor}

O autor agradece o apoio financeiro da Universidade Paranaense (UNIPAR) para a realização desta pesquisa.

Recebido em: 31/05/2019

Aceito em: 02/04/2020 